\title{
Characterization of additive cost sharing methods*
}

\author{
Hervé Moulin and Rakesh Vohra
}

April 2003

\begin{abstract}
The set of additive cost sharing methods when individual demands are integer valued and cost shares are non negative, is characterized by its extreme points and by a network flow representation. The extreme methods allocate costs incrementally along a chain of demand vectors independent of the cost function. The result generalizes Wang's characterization in that we do not assume the Dummy axiom.

Key words: cost sharing, additivity, network flow, dummy axiom.

JEL Classification numbers: Cr1, D70

Hervé Moulin, Dept of Economics, Rice University, Houston TX 772511892, moulin@rice.edu, Ph 713-348-3312, Fax 713-348-6329

Rakesh Vohra, Dept of Managerial Economics and Decision Sciences, Kellogg School of Management, Northwestern University, Evanston IL 60208, r-vohra@nwu.edu, Ph 847-491-5428, Fax 847-467-1220.
\end{abstract}

* Acknowledgments: We are grateful to Yves Sprumont and YunTong Wang for stimulating conversations. This research is supported by the NSF under grants SES-0112032 (Moulin) and ITR IIS-0121678 (Vohra). 


\section{Cost sharing with discrete demands}

We consider the familiar model where each one of a finite set of agents $N$ demands a certain quantity $x_{i}$ of an idiosyncratic commodity $i, i \in N$. The demand profile $x=\left(x_{i}\right)_{i \in N}$ generates joint costs $C(x)$. A cost sharing method divides $C(x)$ among $N$, based on $x$ and the entire cost function $C$.

The axiomatic treatment of this problem has a long history in cooperative game theory and a variety of applications. A survey treatment with extensive references is Moulin [2002].

We examine the discrete version of this model where for all $i, x_{i}$ is an integer: each commodity comes in indivisible units. The discrete model, a recent addition to the literature (Moulin [1995], de Nouweland et al. [1995], Sprumont [2000]), is topologically simpler than its more familiar continuous counterpart where $x_{i}$ is a non-negative real number. It also contains the classic cooperative game model as the special case where $x_{i}$ can only be zero or one.

The property that cost shares depend additively upon the cost function $C$ is the key to most of the axiomatic results to date, beginning with Shapley's seminal paper (Shapley [1953]). Here we characterize the extreme points of the set of additive cost sharing methods. Our result generalizes a similar characterization for the set of additive methods meeting the Dummy axiom due to Wang [1999].

The Dummy property is the central equity postulate of Shapley's theory. It conveys the familiar idea of no cross-subsidization: individual agents should not pay for costs for which they are not responsible. An alternative principle is that equal demands should be charged equally, irrespective of asymmetries of the cost function. The two principles are not compatible, and both are commonly used: e.g., rural and urban mail are charged identically, despite the difference in their respective costs; on the other hand, international mail costs more than domestic mail. See Moulin and Sprumont [2002] for an extensive discussion of these two alternative principles in the context of additive cost sharing methods. Our theorem is the basis for a more general theory of additive cost sharing, one where the Dummy axiom is not automatically satisfied.

A corollary of our results is a variant of Wang's theorem, where the Dummy axiom is replaced by the apparently weaker property that we call Non-Dummy: if all agents but one are dummies, the non-dummy agent bears all the costs. We show that Dummy and Non-Dummy are equivalent for additive cost sharing methods. 


\section{The model and main result}

We fix the finite set $N$ of agents. A demand profile $x$ is an element of $\mathbb{N}^{N}$, where $\mathbb{N}=\{0,1, \ldots\}$. The demand profile $e^{i}$ is the $\mathrm{i}$-th coordinate vector. A cost function $C$ maps $\mathbb{N}^{N}$ into $\mathbb{R}_{+}^{N}$, is non-decreasing and $C(0)=0$. We write $\mathcal{C}$ for the set of such functions.

A cost sharing problem is a pair $(x, C) \in \mathbb{N}^{N} \times \mathcal{C}$. A solution of this problem is a profile of cost shares $y \in \mathbb{R}_{+}^{N}$, such that $\sum_{N} y_{i}=C(x)$. A cost sharing method $\varphi$ maps $\mathbb{N}^{N} \times \mathcal{C}$ into $\mathbb{R}_{+}^{N}$ : it picks a solution for every cost-sharing (in short, c.s.) problem. The following property of c. s. methods is the subject of this paper:

Additivity: for all $C^{1}, C^{2} \in \mathcal{C}, x \in \mathbb{N}^{N}: \varphi\left(x, C^{1}\right)+\varphi\left(x, C^{2}\right)=\varphi\left(x, C^{1}+C^{2}\right)$

We denote by $\Phi$ the set of additive cost sharing methods.

For any c. s. method $\varphi$ and demand profile $x$, we write $\varphi(x)$ for the restriction of $\varphi$ to the problems $(x, C)$ : thus $\varphi(x)$ maps $\mathcal{C}$ into $\mathbb{R}_{+}^{N}$. Denote $\Phi(x)$ to be the set of additive $x$-restricted methods. Clearly the method $\varphi$ belongs to $\Phi$ if and only if $\varphi(x)$ is in $\Phi(x)$ for all $x \in \mathbb{N}^{N}$. In the sequel we fix an arbitrary demand profile $x \in \mathbb{N}^{N}$, and describe the extreme points of $\Phi(x)$. Note that $\Phi(0)$ contains only one (trivial) method. We assume $x \neq 0$ from now on.

A chain to $x$ (or $x$-chain) is a sequence $0=z^{0} \supsetneqq z^{1} \supsetneqq \ldots \supsetneqq z^{K}=x$ increasing from 0 to $x$. The length $K$ of an $x$-chain varies between 1 and $\sum_{N} x_{i}$. An $x$-chain of length $\sum_{N} x_{i}$ is called a path from 0 to $x$. An $x$-chain is labeled if we attach to each $k, k=1, \ldots, K$, an agent $i_{k} \in N$, with the restriction that two consecutive agents are different: $i_{k} \neq i_{k-1}$ for $k=1, \ldots, K$. We denote a labeled chain as $\gamma=\left(z^{k}, i_{k}\right)_{k=1, \ldots K}$.

Definition: To any labeled $x$-chain $\gamma$, we associate a c. s. method $\varphi^{\gamma}(x)$

as follows: $\varphi^{\gamma}(x)(C)=\sum_{k=1}^{K}\left(C\left(z^{k}\right)-C\left(z^{k-1}\right)\right) \cdot e^{i_{k}}$, for all $C \in C$.

These cost shares are non-negative because $C$ is nondecreasing, and sum to $C(x)$. Additivity is clear. Thus $\varphi^{\gamma}(x)$ is an element of $\Phi(x)$.

The reader can easily verify that the mapping $\gamma \rightarrow \varphi^{\gamma}(x)$ is one-to-one: two distinct labeled $x$-chains yield different cost sharing methods (recall that two consecutive labels are different). The number $\rho(x)$ of labeled $x$-chains is large; one checks easily

$$
\frac{x_{N} !}{x_{1} ! \ldots x_{n} !} \cdot(n-1)^{x_{N}} \leq \rho(x) \leq \frac{x_{N} !}{x_{1} ! \ldots x_{n} !} \cdot n^{x_{N}}, \text { where } x_{N}=\sum_{N} x_{i} .
$$

Theorem 1 Fix $N$ and $x \in \mathbb{N}^{N}$. The cost sharing methods associated with the labeled $x$-chains are precisely the extreme points of $\Phi(x)$. In particular, every $x$-method $\varphi(x) \in \Phi(x)$ can be written as a (possibly non-unique) convex combination of such methods. 
The theorem is a consequence of a network flow representation of additive cost sharing methods. We use the following notations: $] 0, x]=\left\{z \in \mathbb{N}^{N} \mid 0 \supsetneqq\right.$ $z \leq x\},] 0, x\left[=\left\{z \in \mathbb{N}^{N} \mid 0 \supsetneqq z \supsetneqq x\right\},[w, x]=\left\{z \in \mathbb{N}^{N} \mid w \leq z \leq x\right\}, N(z)=\right.$ $\left\{i \in N \mid z_{i}>0\right\}, P(z)=\left\{i \in N \mid z_{i}<x_{i}\right\}$, and $\partial_{i} C(z)=C(z)-C\left(z-e^{i}\right)$ for all $i \in N(z)$. Finally, we write the $N$-unit simplex as $\Delta(1)=\left\{\delta \in \mathbb{R}_{+}^{N} \mid\right.$ $\left.\sum_{N} \delta_{i}=1\right\}$.

To each element $z \in[0, x]$ we associate a vertex. Between each pair $z$, $z+e^{i} \in[0, x]$ where $i \in P(z)$, we insert a directed edge from $z$ to $z+e^{i}$ of unlimited capacity. Let $G_{x}$ denote this network. Let $f\left(z, z+e^{i}\right)$ be the flow through the directed edge $\left(z, z+e^{i}\right)$. A flow of a single unit from 0 to $x$ in this network is any solution to the following system:

$$
\begin{gathered}
\sum_{i \in N(x)} f\left(0, e^{i}\right)=\sum_{i \in N(x)} f\left(x-e^{i}, x\right)=1 \\
\left.\sum_{i \in N(z)} f\left(z-e^{i}, z\right)=\sum_{i \in P(z)} f\left(z, z+e^{i}\right) \text { for all } z \in\right] 0, x[ \\
f\left(z, z+e^{i}\right) \geq 0 \text { for all } z \in[0, x], i \in P(z)
\end{gathered}
$$

It is well known that the extreme points of this polyhedron will be the incidence vectors of paths from 0 to $x$.

Given a feasible unit flow, we can share it amongst the agents by specifying how much of the flow through each edge is allocated to each agent. To each directed edge $\left(z, z+e^{i}\right)$, we associate a vector $s\left(z, z+e^{i}\right) \in \Delta(1)$. The $r^{t h}$ component of $s\left(z, z+e^{i}\right)$ is the fraction of the flow through $\left(z, z+e^{i}\right)$ assigned to agent $r$.

\section{Theorem 2 Flow representation}

Given a unit flow $f$ to $x$ and a collection $s\left(z, z+e^{i}\right) \in \Delta(1)$ for each directed edge $\left(z, z+e^{i}\right)$, the following equation defines a method $\varphi(x)$ in $\Phi(x)$ : $\varphi(x)(C)=\sum_{z \in] 0, x]} \sum_{i \in N(z)} \partial_{i} C(z) \cdot f\left(z-e^{i}, z\right) \cdot s\left(z-e^{i}, z\right)$, for all $C \in \mathcal{C}$.

Conversely, any method $\varphi(x)$ in $\Phi(x)$ can be represented (in at least one way) by a pair $(s, f)$ as above.

To derive Theorem 1 from Theorem 2, observe that a method $\varphi(x)$ represented by $(s, f)$ is a convex combination of methods represented by $\left(s^{\prime}, f^{\prime}\right)$, where the range of $s^{\prime}$ consists of coordinate vectors and $f^{\prime}$ is the incidence vector of a path $y^{t}, t=1, . ., \sum_{N} x_{i}$ from 0 to $x$. Set $s^{\prime}\left(y^{t}, y^{t+1}\right)=e^{i(t)}$ and let $\left[0, t_{1}\right],\left[t_{1}+1, t_{2}\right], . .,\left[t_{K-1}+1, t_{K}\right]$ be the coarsest partition of $\left[0, \sum_{N} x_{i}\right]$ such that $e^{i(t)}$ is constant in each interval. Write $z^{k}=y^{t_{k}}$ and $i_{k}=i\left(t_{k}\right)$ to define a labeled chain $\gamma$ such that $\varphi^{\gamma}(x)$ is precisely the method represented by $\left(s^{\prime}, f^{\prime}\right)$. Thus Theorem 2 implies that any $\varphi(x) \in \Phi(x)$ is a convex combination of methods $\varphi^{\gamma}(x)$. The other statement of Theorem 1 is that each method $\varphi^{\gamma}(x)$ is an extreme point of $\Phi(x)$. It is proven in subsection 4.2. 


\section{Application: Dummy and Non-Dummy}

Dividing costs in fixed proportions, irrespective of demands and cost asymmetries, is a simple method in $\Phi: \varphi(x, C)=C(x) \cdot \delta$, where $\delta$ is independent of $x$ and $C$. More responsive to the size of individual demands, the familiar proportional method, $\varphi(x, C)=\frac{C(x)}{\sum x_{i}} \cdot x$, also in $\Phi$, is equally oblivious to cost asymmetries. The Dummy axiom eliminates the two methods above.

Dummy: for all $i \in N, C \in \mathcal{C},\left\{\partial_{i} C(z)=0\right.$ for all $\left.z\right\} \Longrightarrow\left\{\varphi_{i}(x, C)=0\right.$ for all $x\}$.

If serving agent $i$ 's demand is free, Dummy says that $i$ should pay nothing. The next axiom is a weaker requirement: if all but one agent is "free," the remaining agent pays the bill.

Non-Dummy: for all $i \in N, C \in \mathcal{C},\left\{\partial_{j} C(z)=0\right.$ for all $j \in N \backslash i$, all $z\} \Longrightarrow\left\{\varphi_{i}(x, C)=C(x)\right\}$.

We prove that under Additivity, the properties Dummy and Non-Dummy are equivalent. Fix an arbitrary $x$-restricted method $\varphi(x)$ in $\Phi(x)$ meeting Non-Dummy, and check it meets Dummy as well. By Theorem $1, \varphi(x)$ is a convex combination of methods $\varphi^{\gamma}(x)$ associated with some labeled $x$-chains $\gamma$. By definition of cost sharing methods, the range $\varphi^{\gamma}(x)(\mathcal{C})$ is contained in the simplex $\Delta=\left\{y \in \mathbb{R}_{+}^{N} \mid \sum_{N} y_{i}=C(x)\right\}$. For any cost function $C$ where $i$ is the only non-dummy agent, we have $\varphi(x, C)=C(x) \cdot e^{i}$. As $C(x) \cdot e^{i}$ is an extreme point of $\Delta$ it follows that each method $\varphi^{\gamma}(x)$ meets Non-Dummy.

Set $\gamma=\left(z^{k}, i_{k}\right)$ and examine the consequences of Non-Dummy on these two sequences. Suppose $z_{j}^{k}>z_{j}^{k-1}$ for some $j \in N$ and consider the cost function $C, C(z)=1$ if $z_{j} \geq z_{j}^{k}, C(z)=0$ otherwise. Non-Dummy implies $\varphi^{\gamma}(x)(C)=e^{j}$ and the definition of $\varphi^{\gamma}$ gives $\varphi^{\gamma}(x)(C)=e^{i_{k}}$. Thus $z^{k}-z^{k-1}$ is borne by $e^{i_{k}}$ for all $k=1, \ldots, K$. Equivalently, $\varphi$ is described by a sequence $\left(\lambda_{k}, i_{k}\right)$ where $\lambda_{k} \in \mathbb{N} \backslash\{0\}$ and $i_{k} \in N$; and the chain $z^{k}$ is defined inductively by $z^{k}=z^{k-1}+\lambda_{k} \cdot e^{i_{k}}$.

Now fix $C \in \mathcal{C}$ for which a certain agent $i$ is a dummy. In the sum defining $\varphi_{i}^{\gamma}(x)(C)$, all terms where $i_{k} \neq i$ are zero; and when $i_{k}=i$, the difference $C\left(z^{k}\right)-C\left(z^{k-1}\right)$ is zero as well. Thus $\varphi^{\gamma}(x)$ satisfies Dummy.

The $x$-methods $\varphi^{\gamma}(x)$ just described are called path generated $x$-methods in Wang [1999]. Indeed the labeled chain $\gamma$ is described by an increasing path from 0 to $x$ in which each increment raises exactly one coordinate; the corresponding cost increment is charged to this very agent.

Corollary 3 Role of Dummy and Non-Dummy

Given a method $\varphi(x)$ in $\Phi(x)$, the three following properties are equivalent:

i) $\varphi(x)$ meets Dummy

ii) $\varphi(x)$ meets Non-Dummy

iii) $\varphi(x)$ is a convex combination of path generated methods.

Wang [1999] proves the equivalence of i) and iii). It is easy to check that under the assumptions of Corollary 3, the flow representation in Theorem 2 is unique. 
The special case where $x_{i}=1$ for all $i$ is the classic cooperative game model. Corollary 3 implies a simple characterization of the random order values (Weber [1988]). In the class of monotonic games, they are the only values meeting Additivity, Non-Dummy, and non-negative shares.

\section{Proofs}

\subsection{Notations and preliminary results}

We fix $x$ throughout, and write an element of $\Phi(x)$ simply as $\varphi$. The first observation is that $\varphi(C)$ only depends upon the restriction of $C$ to $[0, x]$. For any two $C^{1}, C^{2}$ in $\mathcal{C}, C^{1}=C^{2}$ on $[0, x]$ implies $\varphi\left(C^{1}\right)=\varphi\left(C^{2}\right)$. This property is called Independence of Irrelevant Costs and is proved in Lemma 1 of Moulin [1995], to which we refer the reader for a proof.

Denote by $\mathcal{C}(x)$ the subset of $\mathbb{R}_{+}^{[0, x]}$ made of non-decreasing functions such that $C(0)=0$ and $C(x)>0$. We can now view $\varphi$ as an additive mapping from $\mathcal{C}(x)$ into $\mathbb{R}_{+}^{N}$. Define $\mathcal{D}=\{D \in \mathcal{C}(x) \mid D(z)=0$ or 1 , for all $z \in[0, x]\}$. In particular, $D(0)=0$ and $D(x)=1$. As this causes no confusion, we identify $D$ and $D^{-1}(1)$, so that $\mathcal{D}$ is the set of upper comprehensive non-empty subsets of ]0, $x$. Note that $\varphi$ maps $\mathcal{D}$ into the simplex $\Delta(1)$.

Additivity implies that $\varphi$ is modular on $\mathcal{D}: \varphi\left(D^{1}\right)+\varphi\left(D^{2}\right)=\varphi\left(D^{1} \cup D^{2}\right)+$ $\varphi\left(D^{1} \cap D^{2}\right)$ for all $D^{1}, D^{2} \in \mathcal{D}$. A modular function on a finite lattice takes the following form:

$$
\varphi(D)=\sum_{D} \lambda(z) \quad \text { for all } D \in \mathcal{D}
$$

where $\lambda(z) \in \mathbb{R}^{N}$ for all $\left.\left.z \in\right] 0, x\right]$. Therefore the mapping $z \rightarrow \lambda(z)$ is a solution of the following linear system:

$$
\sum_{z \in D} \lambda(z) \in \Delta(1) \quad \text { for all } D \in \mathcal{D} .
$$

Conversely, to each mapping $\lambda \in\left(\mathbb{R}^{N}\right)^{10, x]}$ satisfying the system (1), we associate a unique $\varphi \in \Phi(x)$ as follows. Each cost function $C \in \mathcal{C}(x)$ has a unique decomposition

$$
C=\sum_{1}^{K} \mu_{k} \cdot D_{k} \text { where } \mu_{k}>0, D_{k} \in \mathcal{D} \text {, and } D_{1} \supsetneqq D_{2} \ldots \supsetneqq D_{K}
$$

(To prove this claim, notice that the range of $C$ must be $0, \mu_{1}, \mu_{1}+\mu_{2}, \ldots$ ). Then we define $\varphi(C)=\sum_{1}^{K} \mu_{k} \cdot \varphi\left(D_{k}\right)$. It is left to the reader to check that the mappings $\varphi \longrightarrow \lambda$ and $\lambda \longrightarrow \varphi$ just constructed, form a linear isomorphism of $\Phi(x)$ into the subset $\Lambda$ of $\left(\mathbb{R}^{N}\right)^{10, x]}$ defined by (1). 


\subsection{Extremality of $\varphi^{\gamma}$ in $\Phi(x)$.}

This follows at once from two observations. For all $D, \varphi^{\gamma}(D)$ is a coordinate vector, namely an extreme point of $\Delta(1)$, whereas $\varphi(D) \in \Delta(1)$ for all $D$ and all $\varphi \in \Phi(x)$. Moreover by Step 1, two elements of $\Phi(x)$ that coincide on $\mathcal{D}$ must be equal.

\subsection{Proof of Theorem 3.}

The first statement is clear: for any $(s, f)$ the formula in the theorem defines a method in $\Phi(x)$. Conversely, we pick $\varphi$ in $\Phi(x)$ and derive a flow representation of the corresponding $\lambda \in \Lambda$.

\subsubsection{Step 1}

We show first that there exist non-negative vectors $\mu$, one for each directed edge of $G_{x}$ such that

$$
\begin{gathered}
\left.\lambda(z)=\sum_{r \in N(z)} \mu\left(z-e^{r}, z\right)-\sum_{r \in P(z)} \mu\left(z, z+e^{r}\right) \forall z \in\right] 0, x[ \\
\lambda(x)=\sum_{r \in N(x)} \mu\left(x-e^{r}, x\right) .
\end{gathered}
$$

Restating this componentwise for any $i \in N$ :

$\left.\lambda_{i}(z)=\sum_{r \in N(z)} \mu_{i}\left(z-e^{r}, z\right)-\sum_{r \in P(z)} \mu_{i}\left(z, z+e^{r}\right), \forall z \in\right] 0, x[$

and

$$
\lambda_{i}(x)=\sum_{r \in N(x)} \mu_{i}\left(x-e^{r}, x\right)
$$

where $\lambda_{i}$ and $\mu_{i}$ denotes the $i^{t h}$ component of $\lambda$ and $\mu$ respectively.

For $i$ fixed, we define a network flow problem on $G_{x}$ by the system (2), (3) and (4) as follows:

$\lambda_{i}(0)=-\sum_{r \in N(x)} \mu_{i}\left(0, e^{r}\right)$.

Note that we have introduced a free variable $\lambda_{i}(0)$ which we will set equal to $-\sum_{z \in] 0, x]} \lambda_{i}(z)$. Since $\left.] 0, x\right] \in \mathcal{D},(1)$ implies that $\sum_{z \in] 0, x]} \lambda_{i}(z) \geq 0$ and so $\lambda_{i}(0) \leq 0$. 
Each $\lambda_{i}(z)$ is interpreted as the supply/demand of the vertex $z$ Specifically, if $\lambda_{i}(z)>0$ we say that vertex $z$ demands the amount $\lambda_{i}(z)$; if $\lambda_{i}(z) \leq 0$ we say that vertex $z$ supplies the amount $-\lambda_{i}(z)$. We write $D E$ and $S U$ respectively for the set of demand and supply vertices. Note that $\{0\} \in S U$.

We must prove the existence of a non-negative flow $\mu_{i}\left(z-e^{r}, z\right)$ satisfying the system (2), (3), (4). We derive first a necessary condition for existence. If $z \in D E$ it can only receive flow from vertices in $[0, z]$. Thus there must be enough supply among vertices in $S U \cap[0, z]$ to satisfy this demand. This generalizes to any subset $T$ of demand vertices. We use the notation $\Gamma(T)=\cup_{z \in T}[0, z]$ for any $T \subseteq[0, x]$. A necessary condition for feasibility of system $(2),(3),(4)$ is

$$
\sum_{z \in T} \lambda_{i}(z) \leq \sum_{w \in \Gamma(T) \cap S U}-\lambda_{i}(w) \text { for all } T \subseteq D E .
$$

We claim that the condition is sufficient as well. Consider the bipartite graph on $S U \times D E$ where $z \in S U$ is connected to $w \in D E$ iff $z \leq w$. By Hall's marriage theorem, system (5) implies the existence of a non-negative flow $g(z, w)$ such that $-\lambda_{i}(z)=\sum_{w \in \Gamma^{-1}(z) \cap D E} g(z, w)$ for all $z \in S U$, and $\lambda_{i}(w)=\sum_{z \in \Gamma(w) \cap S U} g(z, w)$ for all $w \in D E$. If we implement each flow of size $g(z, w)$ between $z$ and $w$ by a non negative flow on $G_{x}$ from $z$ to $w$, the sum of these flows is a solution $\mu$ of system (2), (3), (4), proving the claim.

We now prove that the inequalities in (5) hold. Suppose not. Then there is a $T \subseteq D E$ such that $\sum_{z \in T} \lambda_{i}(z)>\sum_{w \in \Gamma(T) \cap S U}-\lambda_{i}(w)$. As $\lambda_{i}(z) \geq 0$ for all $z \in D E$, we get

$$
\sum_{z \in \Gamma(T) \cap D E} \lambda_{i}(z) \geq \sum_{z \in T} \lambda_{i}(z)>-\lambda_{i}(0)-\sum_{w \in \Gamma(T) \cap S U, w \neq 0} \lambda_{i}(w) .
$$

Recalling our choice of $\lambda_{i}(0)$ and rearranging we obtain

$$
\begin{aligned}
0 & >-\left[\sum_{z \in \Gamma(T) \cap D E} \lambda_{i}(z)+\sum_{w \in \Gamma(T) \cap S U, w \neq 0} \lambda_{i}(w)\right]-\lambda_{i}(0)= \\
& =-\sum_{z \in \Gamma(T) \backslash\{0\}} \lambda_{i}(z)+\sum_{z \in] 0, x]} \lambda_{i}(z)=\sum_{z \notin \Gamma(T)} \lambda_{i}(z) .
\end{aligned}
$$

But the complement of $\Gamma(T)$ is in $\mathcal{D}$ and so by (1) $\sum_{z \notin \Gamma(T)} \lambda_{i}(z) \geq 0$. This is the desired contradiction.

\subsubsection{Step 2: construction of $(\mathrm{s}, \mathrm{f})$}

For each directed edge $\left(z-e^{r}, z\right)$ let $f\left(z-e^{r}, z\right)=\sum_{i \in N} \mu_{i}\left(z-e^{r}, z\right)$. If this sum is positive, we set for each $i \in N$

$$
s_{i}\left(z-e^{r}, z\right)=\frac{\mu_{i}\left(z-e^{r}, z\right)}{\sum_{k \in N} \mu_{k}\left(z-e^{r}, z\right)}
$$

Clearly $s\left(z-e^{r}, z\right) \in \Delta(1)$ and

$$
\mu\left(z-e^{r}, z\right)=f\left(z-e^{r}, z\right) s\left(z-e^{r}, z\right) .
$$


If $\mu\left(z-e^{r}, z\right)=0$, we have $f\left(z-e^{r}, z\right)=0$ and we choose $s\left(z-e^{r}, z\right)$ arbitrarily in $\Delta(1)$. Thus for all $i \in N$, and $z \in] 0, x]$

$$
\lambda_{i}(z)=\sum_{r \in N(z)} f\left(z-e^{r}, z\right) s_{i}\left(z-e^{r}, z\right)-\sum_{r \in P(z)} f\left(z, z+e^{r}\right) s_{i}\left(z, z+e^{r}\right) .
$$

We show that $f$ constitutes a unit flow from 0 to $x$ in $G_{x}$. First we verify that $\sum_{r \in N(x)} f\left(x-e^{r}, x\right)=1$, that is one unit of flow enters vertex $x$. Since $\{x\} \in \mathcal{D},(1)$ implies $\sum_{i \in N} \lambda_{i}(x)=1$. Hence

$$
\begin{aligned}
1 & =\sum_{i \in N} \lambda_{i}(x)=\sum_{i \in N} \sum_{r \in N(x)} f\left(x-e^{r}, x\right) s_{i}\left(x-e^{r}, x\right) \\
& =\sum_{r \in N(x)} f\left(x-e^{r}, x\right)\left[\sum_{i \in N} s_{i}\left(x-e^{r}, x\right)\right]=\sum_{r \in N(x)} f\left(x-e^{r}, x\right) .
\end{aligned}
$$

A similar argument yields for all $z \in] 0, x]$

$$
\sum_{i \in N} \lambda_{i}(z)=\sum_{r \in N(z)} f\left(z-e^{r}, z\right)-\sum_{r \in P(z)} f\left(z, z+e^{r}\right) .
$$

We show $\sum_{i \in N} \lambda_{i}(z)=0$, namely conservation of flow at all vertices $\left.z \in\right] 0, x[$. Observe that $[z, x]$ and $\cup_{i \in P(z)}\left[z+e^{i}, x\right]$ are in $\mathcal{D}$ and apply (1) to these two sets:

$$
\begin{gathered}
\sum_{i \in N} \sum_{w \in[z, x]} \lambda_{i}(w)=1, \\
-\sum_{i \in N} \sum_{w \in \cup_{r \in P(z)}\left[z+e^{r}, x\right]} \lambda_{i}(w)=-1 .
\end{gathered}
$$

Adding the two yields the desired equality. Given a flow into $x$ of 1 unit and flow conservation at all $z \in] 0, x\left[\right.$, feasibility of (2), (3) and (4) implies $\sum_{N} \lambda_{i}(0)=-1$ hence $\sum_{r \in N(x)} f\left(0, e^{r}\right)=1$. This establishes the claim that $f$ is a unit flow to $x$.

\subsubsection{Step 3}

It remains to show that the method $\varphi$ is represented by the pair $(s, f)$. Choose any $D \in \mathcal{D}$. Denote by $\partial(D)$ the 'boundary' of the set $D$, namely the set of $z \in D$ such that $z-e^{i} \notin D$ for at least one $i \in N(z)$. In terms of the network $G_{x}$ this is the set of vertices in $D$ with at least one neighbor outside $D$. Then

$$
\begin{aligned}
\varphi(D) & =\sum_{z \in D} \lambda(z)=\sum_{z \in D}\left\{\sum_{r \in N(z)} \mu\left(z-e^{r}, z\right)-\sum_{r \in P(z)} \mu\left(z, z+e^{r}\right)\right\} \\
& =\sum_{z \in \partial(D)} \sum_{r: z-e^{r} \notin D} \mu\left(z-e^{r}, z\right) .
\end{aligned}
$$


The last equality follows from the fact that when we add up a collection of flow balance conditions over the vertices of a comprehensive set (i.e. flow in minus flow out) the intermediate terms cancel leaving only the total flow into the set. Viewing $D \in \mathcal{D}$ as a $0-1$ cost function, it is easy to see that $\partial_{i} D(z)=1$ if and only if $z \in \partial(D)$ and $z-e^{i} \notin D$. Hence

$\varphi(D)=\sum_{z \in \partial(D)} \sum_{r: z-e^{i} \notin D} \mu\left(z-e^{r}, z\right)=\sum_{z \in] 0, x]} \sum_{r \in N(z)} \partial_{r} D(z) \cdot f\left(z-e^{r}, z\right) \cdot s\left(z-e^{r}, z\right)$.

This is the desired equality for $D \in \mathcal{D}$. The case of an arbitrary $C \in \mathcal{C}$ follows by additivity of $\varphi$.

\section{References}

[1] Moulin, H., (1995), "On Additive Methods to Share Joint Costs," Japanese Economic Review, 46, 4, 303-332.

[2] Moulin, H., (2002), "Axiomatic Cost and Surplus-Sharing," Chapter 6 of the Handbook of Social Choice and Welfare, K. Arrow, A. Sen and K. Suzumura, Editors, North-Holland.

[3] Moulin, H. and Sprumont, Y., (2002), "Responsibility and crosssubsidization in cost sharing," mimeo, Rice University.

[4] de Nouweland, A. and Tijs, S., (1995), "Cores and related solution concepts for multi-choice games," Mathematical Methods of Operations Research 41:289-311.

[5] Shapley, L., (1953), "A value for n-person games," in: H.W. Kuhn and A.W. Tucker, eds., Contributions to the Theory of Games II, Annals of Mathematics Studies 28:307-317.

[6] Sprumont, Y, (2000), "Coherent cost-sharing," Games and Economic Behavior 33:126-144.

[7] Wang, Y.T., (1999), "The additivity and dummy axioms in the discrete cost sharing model," Economic Letters 64:187-192.

[8] Weber, R., (1988), "Probabilistic values for games," in: A. Roth, ed., The Shapley Value (Cambridge University Press, Cambridge) pp. 101-119. 